F. Carli MD MPHIL FRCA,

K. Klubien MBCHB FRCA

\section{Thoracic epidurals: is analgesia all we want?}

pain-free postoperative period, although an important humanitarian gesture does not automatically lead to improved postoperative outcome. ${ }^{15}$ Unfortunately, this study failed to demonstrate any difference between the two groups in the numbers of patients in whom the trachea was extubated at four hours. No other outcome measurements were assessed. This was a missed opportunity in an otherwise well designed study which should still serve to encourage anesthesiologists to move cephalad in their approach to the epidural space in order to improve the comfort of their patients.

In our quest for excellent analgesia after thoracoabdominal surgery the thoracic epidural approach using a mixture of local anesthetic and opioid has the most favourable profile. To improve perioperative outcome the other advantages of thoracic epidural anesthesia (cardiovascular, respiratory, gastrointestinal, metabolic) must be utilized and integrated into multimodal recovery programs aimed at optimizing outcome after surgery.

Thoracic epidural anesthesia (TEA) with local anesthetic has well documented effects on the cardiovascular system and should, theoretically, influence cardiac morbidity, the primary cause of death after anesthesia and surgery. The postoperative period, however, has not been optimally controlled or standardized in most outcome studies to date. By selective blockade of cardiac sympathetic innervation $\left(T_{1-5}\right)$ TEA improves the balance of oxygen supply and demand in the ischemic myocardium. Conversely, lumbar epidural anesthesia (LEA), which less commonly extends sufficiently cephalad to produce thoracic sympathectomy, may result in increased myocardial ischemia in patients with coronary artery disease. ${ }^{16}$ In addition, LEA is associated with reflex arterial dilatation and vagally mediated bradycardia (Bezold Jarisch reflex) more frequently than TEA due to the extent of the sympathetic blockade. ${ }^{17}$ The hemodynamic stability afforded by TEA, coupled with a reduced stress response, should provide an improved outcome if the tendency to hypotension is controlled. 
Studies of thoracic anesthesia in patients undergoing cardiac surgery demonstrate better hemodynamic stability, earlier extubation, better pain relief and postoperative respiratory function and a reduction of the catecholamine response. ${ }^{17}$

A recently published meta-analysis confirmed that postoperative epidural pain control decreased the incidence of pulmonary morbidity. ${ }^{18}$ Opioids administered by the thoracic epidural route were found to confer no advantage over the lumbar approach. Epidural local anestherics, however, attenuated the pulmonary dysfunction observed after upper abdominal surgery ${ }^{19}$ not only by abolishing pain both at rest and with activity but by a specific action of local anesthetics on diaphragmatic function. ${ }^{20}$ Improved respiratory outcome would therefore be expected with thoracic local anesthetic and opioid mixtures but due to a paucity of studies this has yet to be confirmed.

Effective pain control with epidural local anesthetic promotes recovery of intestinal function ${ }^{9,14}$ because the associated sympathetic intestinal nerve block increases bowel motility. Administering local anesthetics at thoracic levels reduces the dose required to achieve pain control and the opioid sparing effect limits undesirable side effects of nausea and vomiting, allowing carlier resumption of oral nutrition. The limited segmental spread of the thoracic epidural analgesia also minimizes motor blockade thus facilitating early mobilization. ${ }^{21}$

The classic catabolic response to operation (the surgical stress response), a key pathogenic factor in postoperative morbidity, is attenuated by blocking the afferent neural stimulus with epidural local anesthetic. ${ }^{22}$ Extensive epidural anesthesia $\left(\mathrm{T}_{4}-\mathrm{S}_{5}\right)$ is easily achieved by the thoracic route without approaching toxic local anesthetic concentrations and, when instituted before surgery and followed by epidural analgesia with local anesthetic, results in the greatest observed reduction of the perioperative stress response. ${ }^{16}$ Kehlet has hypothesized that, with multimodal interventions including the use of epidural anesthesia to control the surgical stress response and prolonged effective pain relief, the undesirable surgical sequelae and overall costs may be reduced. ${ }^{22}$ This hypothesis was tested in patients undergoing abdominothoracic esophagectomy with thoracic epidural local anesthetic and opioid administration over a prolonged perioperative period. Significant improvements in outcome (improved nitrogen balance, earlier extubation and mobilization, earlier discharge from ICU) were demonstrated in those following a multimodal approach. ${ }^{23}$ This study. draws attention to the importance of meticulous documen- tation of adequate segmental blockade of the entire surgical field throughout the entire experimental period. In addition, it addresses the benefits of a global approach to perioperative outcome.

With the benefits of thoracic epidurals now clearly demonstrated the safety of this procedure must be questioned. Does thoracic catheterization pose a greater risk to the patient than the lumbar approach? The most feared complication of epidural nerve blocks, permanent neurological damage, has received much attention in the literature ${ }^{24-28}$ although the true prevalence of neurological sequelae solely attributable to anesthetic causes remains difficult to estimate ${ }^{27}$ and the specific contribution of thoracic epidurals to this figure has rarely been addressed. The risk of spinal hematoma is less than 1 in 100,000 in most series ${ }^{24}$ although a recent study ${ }^{26}$ reported a much higher incidence of permanent neurological deficits of 1 in 4,612 . The two cases reported were both lumbar epidurals and the ratio of lumbar to thoracic epidurals in the series was not documented. In a study looking specifically at thoracic epidurals no permanent neurological sequelae were reported in 4,185 patients. ${ }^{28}$

Neurological injuries caused by neuraxial blockade fall into two main etiological groups: 1) those arising at the induction of blockade and 2) those related to shortcomings in postoperative surveillance. ${ }^{27}$ Accidental puncture of the underlying cord at block induction, although anatomically more likely at thoracic levels, can still occur at high lumbar levels and a technique that minimizes this risk should be adopted for any epidural. If performed in the conscious patient trauma to the underlying neural structures will elicit pain that should warn the operator to stop immediately. Risk factors for neurological complications have been well identified ${ }^{24}$ and should be carefully considered before embarking upon neuraxial block whether it be lumbar or thoracic.

Vigilant monitoring of the postoperative period is essential for early recognition of the signs of spinal hematoma or abscess. Low dose local anesthetic and opioid mixtures in thoracic epidurals should cause minimal leg weakness offering no impedance to the early recognition of leg weakness associated with spinal cord compression. To exploit the full benefits from thoracic epidural analgesia, pain relief must be tailored to the requirements of each individual patient with monitoring of sensory and motor blockade and side effects (sedation, hypotension and respiratory depression) throughout the prolonged period of postoperative analgesia. A commitment to on-going staff education in the care and monitoring of patients following surgery is essential and the benefits to the patient will rapidly become evident. 
In summary, the thoracic epidural approach using local anesthetic and opioid is certainly desirable not only for its excellent analgesia but for its pivotal role in multimodal treatment programs which should lead to important advances in recovery from surgery and reductions in overall morbidity and cost. Testing this hypothesis is the challenge which must now be embraced by anesthesiologists in conjunction with all health care providers involved in perioperative care.

\section{Péridurale thoracique : tout ce que nous voulons c'est l'analgésie?}

L'anesthésie et l'analgésie péridurales thoraciques sont pratiquées largement et avec enthousiasme par un grand nombre d'anesthésiologistes lors d'interventions chirurgicales thoraciques et abdominales. Cependant, beaucoup de praticiens les considèrent toujours comme des techniques associées à des risques qui ne justifient pas leur emploi, surtout quand on sait qu'il existe d'autres choix analgésiques attrayants. Ils seraient, toutefois, peu nombreux à remettre en question l'efficacité de la voie péridurale lombaire que ce soit pour l'anesthésie peropératoire ou pour le soulagement de la douleur postopératoire. Les opioïdes périduraux procurent un soulagement supérieur de la douleur que les opioïdes généraux. ${ }^{1}$ On a montré qu'ils réduisent le coût et la longueur du séjour hospitalier ${ }^{2}$ et qu'ils ont acquis une grande popularité malgré leurs effets secondaires : nausée, prurit, rétention urinaire et dépression respiratoire potentielle. ${ }^{3} \mathrm{La}$ morphine ne présente pas d'avantages quand elle est administrée par voie thoracique comparée à la voie lombaire ${ }^{4}$ quoique les opioïdes lipophiles fentanyl et sufentanil, avec leur rapidité d'action et leur faible potentiel de dépression respiratoire tardive, semblent être plus efficaces de façon marginale à la suite d'une thoracotomie s'ils sont administrés au niveau thoracique comparé aux voies lombaire ou intraveineuse..$^{5-7}$ Les opioïdes périduraux, toutefois, ne fournissent pas une analgésie optimale quand les patients tentent de se déplacer après une opération. ${ }^{8}$ Plus récemment, on a démontré que la synergie de la combinaison d'anesthésique local et d'opioïde dans l'espace épidural thoracique, en créant une zone limitée d'analgésie ciblée à la région segmentaire de l'incision, produit une meilleure analgésie au retour de l'activité ${ }^{9}$ et réduit les effets défavorables reliés à la dose de tout agent utilisé seul. ${ }^{10}$ De nombreuses études ont montré l'efficacité analgésique supérieure de perfusions d'anesthésiques locaux et d'opiö̈des au moyen de cathéters périduraux thoraciques., ${ }^{70-13}$ Des agents optimaux, des schémas posologiques et des bénéfices comparés sont maintenant à l'étude.

Kahn et coll. rapportent, dans le présent numéro, une étude de 22 patients qui ont subi une œesophagectomie thoraco-abdominale élective confirmant le soulagement supérieur de la douleur obtenu avec une analgésie péridurale thoracique contrôlée par le patient (APCP) avec du fentanyl et de la bupivacaïne comparée à l'APCP avec de la morphine péridurale lombaire. ${ }^{14}$ Cependant, une période postopératoire sans douleur, quoique geste humanitaire important, ne conduit pas automatiquement à une amélioration des complications postopératoires. ${ }^{15}$ Malheureusement, cette étude ne réussit pas à démontrer de différence entre les deux groupes quant au nombre de patients chez qui la trachée a été extubée quatre heures après l'intervention. Aucune autre mesure des résultats n'a été évaluée. C'est une occasion manquée dans une étude par ailleurs bien conçue qui peut encore servir à encourager les anesthésiologistes à déplacer en direction céphalique leur approche de l'espace péridural dans le but d'améliorer le confort de leurs patients.

Dans notre quête d'une excellente analgésie à la suite d'une intervention thoraco-abdominale, l'approche péridurale thoracique utilisant un mélange d'anesthésique local et d'opioïde présente les caractéristiques les plus favorables. Pour améliorer l'évolution périopératoire, les autres avantages de l'anesthésie péridurale thoracique (cardio-vasculaire, respiratoire, gastro-intestinal, métabolique) doivent être utilisés et intégrés dans un programme multimodal de récupération dont le but est d'optimaliser les résultats postopératoires.

L'anesthésie péridurale thoracique (APT) avec des anesthésiques locaux présente des effets bien documentés sur le système cardio-vasculaire et devrait, en théorie, influencer la morbidité cardiaque, la principale cause de décès à la suite de l'anesthésie et de la chirurgie. La période postopératoire n'a cependant pas été surveillée de façon optimale ou standardisée dans la plupart des études sur les complications jusqu'à maintenant. Par un blocage sélectif de l'innervation cardiaque sympathique, $\left(\mathrm{T}_{1-5}\right)$ l'APT améliore l'équilibre entre l'apport et la demande en oxygène pour le myocarde ischémique. Inversement, l'anesthésie péridurale lombaire (APL), qui présente moins fréquemment une extension céphalique suf- 
fisante pour produire une sympathectomie thoracique, peut provoquer une accentuation de l'ischémie myocardique chez des patients atteints de maladie coronarienne. ${ }^{16}$ En outre, l'APL est associée à une dilatation artérielle réflexe et à une bradycardie à médiation vagale (réflexe de Bezold Jarisch) plus souvent que l'APT à cause de l'étendue du blocage sympathique. ${ }^{17} \mathrm{La}$ stabilité hémodynamique offerte par l'APT, couplée à la réponse réduite au stress, peut donner de meilleurs résultats si la tendance à l'hypotension est contrôlée. Les études sur l'anesthésie thoracique chez des patients qui subissent une intervention cardiaque démontrent une meilleure stabilité hémodynamique, une extubation précoce, un soulagement plus efficace de la douleur, une meilleure fonction respiratoire postopératoire et une réduction de la réponse adrénergique. ${ }^{17}$

Une méta-analyse récemment publiée a confirmé que le contrôle péridural postopératoire de la douleur diminue l'incidence de morbidité pulmonaire. ${ }^{18} \mathrm{On}$ a trouvé que les opioïdes administrés par voie péridurale thoracique n'offrent pas d'avantages par rapport à la voie lombaire. Les anesthésiques locaux périduraux atténuent toutefois le dysfonctionnement pulmonaire après la chirurgie abdominale ${ }^{19}$ non seulement en abolissant la douleur au repos ou en activité, mais aussi par une action spécifique des anesthésiques locaux sur la fonction diaphragmatique. ${ }^{20}$ On devrait alors s'attendre à l'amélioration des complications respiratoires avec les mélanges d'anesthésique local et d'opioïde au niveau thoracique, mais à cause du nombre limité d'études, cela reste à vérifier.

Un soulagement efficace de la douleur avec l'anesthésique local péridural favorise la récupération de la fonction intestinale ${ }^{9,14}$ parce que le bloc sympathique intestinal associé accroît la motilité. L'administration d'anesthésiques locaux aux niveaux thoraciques réduit la dose requise pour le soulagement de la douleur et l'effet d'épargne de l'opioïde limite les effets secondaires indésirables tels nausées et vomissements, permettant la reprise plus précoce de l'alimentation orale. L'étendue segmentaire limitée de l'analgésie péridurale thoracique réduit aussi le blocage moteur, facilitant ainsi la mobilisation précoce. ${ }^{21}$

La réponse catabolique classique à l'opération (la réponse au stress chirurgical), un facteur pathogénique clé de la morbidité postopératoire, est atténuée par le blocage du stimulus nerveux afférent avec l'anesthésique local péridural. ${ }^{22}$ L'anesthésie péridurale étendue $\left(\mathrm{T}_{4}\right.$ $\mathrm{S}_{5}$ ) est facilement réalisée par voie thoracique sans s'approcher des concentrations d'anesthésique local toxiques et, quand amorcée avant l'intervention et suivie par l'analgésie péridurale avec un anesthésique local, elle fournit la réduction la plus importante connue de la réponse au stress périopératoire. ${ }^{16}$ Kehlet a émis l'hypothèse qu'avec les méthodes multimodales y compris l'usage de l'anesthésie péridurale pour contrôler la réponse au stress chirurgical et le soulagement efficace et prolongé de la douleur, les séquelles chirurgicales et le coût total peuvent être réduits. ${ }^{22}$ Cette hypothèse a été testée chez des patients qui subissaient une œesophagectomie abdomino-thoracique avec l'administration d'un anesthésique local péridural thoracique et d'opioïde pendant une période périopératoire prolongée. Des améliorations significatives des complications (équilibre azoté amélioré, extubation et mobilisation précoces, départ précoce de'l'USI) ont été démontré chez des patients à la suite d'une intervention multimodale. ${ }^{23}$ Cette étude attire l'attention sur l'importance d'une documentation méticuleuse du blocage segmentaire adéquat du champ chirurgical complet tout au long de la période expérimentale. De plus, l'étude évalue les bénéfices d'une vision globale sur le taux des complications périopératoires.

Maintenant que les bénéfices des méthodes péridurales thoraciques ont été clairement démontrés, la sécurité de l'intervention doit être abordée. Est-ce que le cathétérisme thoracique comporte de plus grands risques que l'approche lombaire? La complication la plus redoutée du blocage péridural, les dommages neurologiques permanents, a retenu énormément l'attention dans la littérature ${ }^{24-28}$ bien que la prévalence réelle de séquelles neurologiques attribuables seulement à l'anesthésique demeure difficile à évaluer ${ }^{27}$ et la contribution spécifique de l'approche thoracique à ce sujet a rarement été abordée. Le risque d'hématome péridural est de moins de 1 sur 100000 dans la plupart des séries ${ }^{24}$ quoiqu'une récente étude ${ }^{26}$ ait rapporté une incidence beaucoup plus élevée de déficit neurologique permanent, soit de 1 sur 4 612. Les deux cas mentionnés concernent deux interventions péridurales lombaires et le ratio de péridurale lombaire par rapport à la péridurale thoracique n'est pas documenté dans les séries. Dans une étude où on a spécifiquement observé les péridurales thoraciques, aucune séquelle neurologique permanente n'a été rapportée chez 4185 patients. ${ }^{28}$

Les atteintes neurologiques causées par un blocage neuraxial se retrouvent dans deux groupes étiologiques principaux : 1) celles qui surviennent à l'induction du bloc et 2) celles qui sont reliées à un défaut de surveillance postopératoire. ${ }^{27}$ Une ponction accidentelle de la dure-mère à l'induction du bloc, bien qu'anatomiquement plus probable au niveau thoracique, peut toujours survenir à des niveaux lombaires supérieurs et une technique qui réduit le risque devrait être adoptée pour 
toute péridurale. Si c'est réalisé chez un patient conscient, le traumatisme des structures neurales sousjacentes vont provoquer une douleur qui avertit immédiatement l'intervenant de stopper l'intervention. Les facteurs de risque de complications neurologiques ont été bien identifiés ${ }^{24}$ et devraient être attentivement considérés avant de procéder au bloc neuraxial, qu'il soit lombaire ou thoracique.

Le monitorage postopératoire observé avec vigilance est essentiel pour reconnaître rapidement les signes d'hématome ou d'abcès périduraux. De faibles doses d'un mélange d'anesthésique local et d'opioïde pour les péridurales thoraciques ne devraient causer qu'une faiblesse minimale des membres inférieurs, sans empêcher l'identification précoce de faiblesse associée à la compression de la moelle épinière. Afin d'exploiter tous les avantages de l'analgésie péridurale thoracique, le soulagement de la douleur doit être ajusté aux besoins de l'individu en surveillant le blocage sensitif et moteur et les effets secondaires (sédation, hypotension et dépression respiratoire) pendant toute la période d'analgésic postopératoire. Un engagement à une mise à jour des connaissances sur les soins et le monitorage des patients après l'intervention chirurgicale et sur les avantages pour le patient deviendra rapidement évident.

En résumé, la méthode péridurale thoracique utilisant l'anesthésique local et l'opioïde est sûrement souhaitable non seulement pour son excellente analgésie, mais aussi pour son rôle capital dans les programmes de traitement multimodal qui devraient amener des améliorations importantes de la récupération postopératoire et des réductions globales de morbidité et de coût. Tester cette hypothèse, c'est le défi que doivent relever les anesthésiologistes en association avec tout le personnel soignant impliqué dans les soins périopératoires.

\section{References}

1 Tsui SL, Chan CS, Chan ASH, Wong SJ, Lam CS, Jones $R D M$. Postoperative analgesia for oesophagael surgery: a comparison of three analgesic regimens. Anaesth Intensive Care 1991; 19: 329-37.

2 Smedstad KG, Beattie WS, Blair WS, Buckley DN. Postoperative pain relief and hospital stay after total esophagectomy. The Clinical Journal of Pain 1992; 8: 149-53.

3 Kavanagh BP, Katz J, Sandler AN. Pain control after thoracic surgery. A review of current techniques. Anesthesiology 1994; 81: 737-59.

4 Fromme GA, Steidl LJ, Danielson DR. Comparison of lumbar and thoracic epidural morphine for relief of postthoracotomy pain. Anesth Analg 1985; 64: 454-5.
5 Salomaki TE, Laitinen JO, Nuutinen LS. A randomized double-blind comparison of epidural versus intravenous fentanyl for analgesia after thoracotomy.

Anesthesiology 1991; 75: 790-95.

6 Guinard JP, Mavrocordatos P, Chiolero R, Carpenter $R L$. A randomized comparison of intravenous versus lumbar and thoracic epidural fentanyl for analgesia after thoracotomy. Anesthesiology 1992; 77: 1108-15.

7 Hansdottir $V$, Woestenborghs $R$, Nordberg $G$. The pharmacokinetics of continuous epidural sufentanyl and bupivacaine infusion after thoracotomy. Anesth Analg 1996; 83: 401-6.

8 Kehlet $H$, Dabl JB. The value of multimodal or balanced analgesia in postoperative pain relief. Anesth Analg 1993; 77: 1048-56.

9 Liu SS, Carpenter RL, Mackey DC, et al. Effects of perioperative analgesic technique on rate of recovery after colon surgery. Anesthesiology 1995; 83: 757-65.

10 Liu S, Angel JM, Owens BD, Carpenter RL, Isabel L. Effects of epidural bupivacaine after thoracotomy. Reg Anesth 1995; 20: 303-10.

11 Watson A, Allen PR. Influence of thoracic epidural analgesia on outcome after resection for esophageal cancer. Surgery $1994 ; 115: 429-32$.

12 Broekema AA, Gielen MJM, Hennis PJ. Postoperative analgesia with continuous epidural sufentanyl and bupivacaine: a prospective study in 614 patients. Anesth Analg 1996; 82: 754-9.

1.3 Wiebalck $A$, Brodner $G$, Van Aken $H$. The effects of adding sufentanyl to bupivacaine for patient-controlled epidural analgesia. Anesth Analg 1997; 85: 124-9.

14 Kabn L, Baxter FJ, Dauphin A, et al. A prospective randomized trial comparing thoracic and lumbar epidural techniques for postthoracoabdominal esophagectomy analgesia. Can J Anesth 1999; 46: 415-22.

15 Moinicbe S, Hjortso NC, Hansen BL, et al. The effect of balanced analgesia on early convalescence after major orthopaedic surgery. Acta Anaesthesiol Scand 1994; 38: 328-35.

16 Liu S, Carpenter $R L$, Neal JM. Epidural anesthesia and analgesia: their role in postoperative outcome. Anesthesiology 1995; 82: 1474-506.

17 Meissner $A, \operatorname{Rolf} N$, Van Aken $H$. Thoracic epidural anesthesia and the patient with heart disease: benefits, risks, and controversies. Anesth Analg 1997; 85: 517-28.

18 Ballantyne JS, Carr DB, de Ferrenti $S$, et al. The comparative effects of postoperative analgesic therapies on pulmonary outcome: cumulative meta-analyses of randomized, controlled trials. Anesth Analg 1998; 86: 598-612.

19 Mankikian B, Cantineau JP, Bertrand JP, Keiffer E, Sartene $R$, Viars $P$. Improvement of diaphragmatic 
function by a thoracic extradural block after abdominal surgery. Anesthesiology 1988; 68: 379-86.

20 Pansard $J L$, Mankikian B, Bertrand $M$, Kieffer $E$, Clergue $F$, Viars $P$. Effects of thoracic extradural block on diaphragmatic electrical activity and contractility after upper abdominal surgery. Anesthesiology 1993; 73: 63-71.

21 Liu SS, Allen HW, Olsson GL. Patient-controlled epidural analgesia with bupivacaine and fentanyl on hospital wards. Anesthesiology 1998; 88: 688-95.

22 Keblet $H$. Multimodal approach to control postoperative pathophysiology and rehabilitation. $\mathrm{Br} J$ Anaesth 1997; 78: 606-17.

23 Brodner G, Pogatzki $E$, Van Aken $H$, et al. A multimodal approach to control postoperative pathophysiology and rehabilitation in patients undergoing abdominothoracic esophagectomy. Anesth Analg 1998; 86: 228-34.

24 Renck $H$. Neurological complications of central nerve blocks. Acta Anaesthesiol Scand 1995; 39: 859-68.

25 Scherer $R$, Schmutzler $M$, Giebler $R$, Erbard J, Stocker $L$, Kox WJ. Complications related to thoracic epidural analgesia: a prospective study in 1071 surgical patients. Acta Anaesthesiol Scand 1993; 37: 370-4.

26 Dahlgren $N$, Tornebrandt $K$. Neurological complications after anaesthesia. A follow-up of 18000 spinal and epidural anaesthetics over three years. Acta Anaesthesiol Scand 1995; 39: 872-80.

27 Bromage PR. Neurological complications of subarachnoid and epidural anaesthesia. Acta Anaesthesiol Scand 1997; 4I: 439-44.

28 Giebler $R M$, Scherer $R U$, Peters $J$. Incidence of neurological complications related to thoracic epidural catheterization. Anesthesiology 1997; 86: 55-63. 